\title{
Labyrinthe
}

13 | 2002

Numéro 13

Document

\section{L'interrogatoire de Tempesta. Un cas de torture dans le duché de Milan à la fin du Xve siècle}

\section{Pierre Savy}

\section{(2) OpenEdition}

Journals

Édition électronique

URL : http://journals.openedition.org/labyrinthe/1511

DOI : $10.4000 /$ labyrinthe.1511

ISSN : 1950-6031

Éditeur

Hermann

Édition imprimée

Date de publication : 15 novembre 2002

Pagination : 69-79

Référence électronique

Pierre Savy, «L'interrogatoire de Tempesta. Un cas de torture dans le duché de Milan à la fin du XV siècle », Labyrinthe [En ligne], 13 | 2002, mis en ligne le 01 mars 2007, consulté le 30 avril 2019. URL : http://journals.openedition.org/labyrinthe/1511; DOI : 10.4000/labyrinthe.1511

Ce document a été généré automatiquement le 30 avril 2019

Propriété intellectuelle 


\title{
Document
}

\section{L'interrogatoire de Tempesta. Un cas de torture dans le duché de Milan à la fin du $X V^{e}$ siècle}

\author{
Pierre Savy
}

«Très illustre seigneur ${ }^{1}$, nous voulons satisfaire à la requête que nous a faite le magnifique $\mathrm{Cicco}^{2}$ de la part de votre Excellence, à propos de ce qui est arrivé à Tempesta de Bobbio $^{3}$, qui fut familier d'armes de votre Seigneurie ; il nous demande de lui dire la pure et entière vérité de la chose. Nous déclarons donc que le magnifique comte Pietro Dal Verme, notre patron, a fait détenir ici, à Pavie ${ }^{4}$, dans les mains du podestat ${ }^{5}$ de votre Seigneurie, ledit Tempesta ; puis, il chargea Matteo Da Monza, son camérier ${ }^{6}$, et d'autres de ses familiers et serviteurs ${ }^{7}$, de le conduire à Castel San Giovanni ${ }^{8}$, dans la région de Plaisance ${ }^{9}$, dans les mains de son châtelain. Et il me chargea, moi, Giacomo Sabbadini, son sénéchal ${ }^{10}$ et maître de maison, le premier jour de juillet de l'année présente, 1470, de le faire aller en ce dit lieu de Castel San Giovanni ; et je devais charger son podestat là-bas d'examiner, de faire examiner et d'interroger Tempesta, avec diligence et subtilité, en suivant les chapitres et l'interrogatoire qui sont écrits ci-dessous ${ }^{11}$; c'est ledit magnifique comte qui me les a donnés.

Aussi, désireux de remplir ma mission, je me rendis ce jour-là, muni de cette commission et de l'interrogatoire, auprès du podestat du bourg de [Castel] San Giovanni ${ }^{12}$. Celui-ci reçut cette commission et l'interrogatoire. Le jour suivant, nous, sénéchal et podestat, qui avions un grand respect et une grande considération pour l'importance de cet interrogatoire et de ces chapitres, nous fîmes vérifier, tous les deux, que la corde du martyre était en ordre. Nous nous demandions si, en cas de besoin, l'on pourrait s'en servir; nous fîmes huiler les poulies, et nous les fîmes préparer par un maître menuisier, si bien qu'il ne nous semblait pas qu'il y eût de danger. Nous fîmes un essai, avant, deux ou trois fois : on faisait tirer en haut depuis le sol à hauteur d'un pas deux hommes à la fois, pour voir si c'était solide. Moi, Niccolò, podestat de ce bourg, en présence du notaire du bourg, je fis chercher Tempesta dans sa prison, puis, le notaire et moi-même, nous l'examinâmes et l'interrogeâmes avec diligence et subtilité, suivant l'interrogatoire et les chapitres, en usant de paroles bonnes et agréables, pour le réduire à dire et avouer la vérité de 
la chose, et nous passâmes peut-être plus de deux heures sur le premier chapitre. Et Tempesta ne disait pas la vérité, en dépit des bonnes paroles que nous usions avec lui. Il me parut, à moi, podestat, qu'il fallait, avant d'en venir à utiliser la corde, user de paroles plus brusques et âpres pour qu'il se mît à trembler et qu'il dît la vérité. Il répondait seulement qu'il n'en pouvait plus ${ }^{13}$, et il blasphémait. Et puisque nous nous apercevions qu'aucune de ces deux techniques ${ }^{14}$ ne parvenait à le faire avouer, à lui faire dire la vérité, j'ordonnai qu'il fût attaché à la corde, et quand il fut attaché, je lui répétai qu'il devait nous dire la vérité car l'on aurait fait montre de miséricorde à son égard et non pas de justice, et je lui répétai de ne pas laisser abîmer sa personne. Celui-ci continuait et persévérait dans son opinion dure et dans sa dureté, et il blasphémait. Je le fis, moi, podestat, tirer en haut depuis le sol, à hauteur d'un pas, presque jusqu'à moitié-corde. Tempesta, alors, voyant cela, cria et dit: «Laissez-moi descendre, je dirai la vérité ! Comme je lui demandais de répondre sur le premier chapitre, qui concernait le fait de votre Seigneurie, il dit je ne sais quelle paroles, il dit qu'il avait parlé et discuté avec messire Obietto ${ }^{15}$, et avec deux autres Fieschi, et comme il me semblait qu'il ne donnait pas pleine satisfaction à l'interrogatoire, je le fis tirer plus haut, jusqu'au plafond, en lui rappelant toutefois qu'il devait avoir compassion de lui-même et qu'il ne devait pas laisser abîmer sa personne. Celui-ci, de plus en plus désespéré, continuait de blasphémer en demandant à voix haute et en criant: "Diable ! emporte-moi et aide-moi ! » Et avec vigueur, plein de furie, il jetait les pieds en l'air pour attraper la corde. Il réclamait toujours de prendre le chemin du diable, et alors qu'il était attaché par les pieds, il semble vraiment que ce fut par jugement divin, la corde se rompit, et lui, il vint frapper le sol en bas avec sa tête, et il frappa sa tête par terre. Il ne parla plus, et moi, Niccolò, je fis aussitôt demander Giacomo, sénéchal, et tous deux, comme nous avions vu et entendu ce qui s'était passé, nous fîmes venir aussitôt deux médecins du bourg, qui arrivèrent vite. On leur raconta ce qui s'était passé, et ils lui firent tous les remèdes possibles pour qu'il revînt à la vie, et quand ils eurent constaté qu'aucun remède ne servait de rien, ils dirent qu'il était mort et qu'il s'était cassé le cou. Nous ordonnâmes donc, avec le châtelain, qu'il ne fût pas enterré jusqu'à ce que l'on eût averti le susdit magnifique comte de ce qui s'était passé. Celui-ci ordonna de l'enterrer. Et pour la vérification et pour la clarté de la vérité elle-même, nous avons, tous les deux, souscrit la présente de notre propre main. Donné à Pavie, le 24 juillet 1470.

Moi, Giacomo Sabbadini, j'ai souscrit de ma propre main ${ }^{16}$.

Moi, Niccolò di Castello de Bologne, j'ai souscrit de ma propre main. »

1 Le document dont nous proposons une traduction nous paraît extraordinaire. Rédigé en italien, à l'exception de quelques passages en latin ${ }^{17}$, il est conservé aux archives d'État de Milan, dans le Carteggio interno du Sforzesco, c'est-à-dire dans la correspondance interne du duché de Milan à l'époque des ducs Sforza ${ }^{18}$. Il raconte une scène de torture infligée à Castel San Giovanni à l'occasion d'un interrogatoire : c'est une torture d'investigation, une "question » finalisée par l'obtention d'aveux, et non un supplice punitif ${ }^{19}$. S'il n'a guère de valeur littéraire - nous avons tâché de respecter le texte jusque dans ses maladresses, par exemple ses répétitions -, sa valeur historique nous paraît certaine. On ne sait rien des hommes qui manient l'instrument, qui torturent directement. En revanche, les deux hommes qui déposent, les auteurs du document, sont nommément désignés. Il s'agit de Giacomo Sabbadini, sénéchal de Pietro Dal Verme à Castel San Giovanni, et de Niccolò di Castello de Bologne, podestat du même. Le premier nous est connu : fils d'Antonio, Giacomo est originaire de Caravate ${ }^{20}$; c'est un fidèle serviteur de Pietro Dal Verme. Il est lui aussi, à l'occasion, désigné comme podestat de Castel San Giovanni, et il représente bien souvent son maître, son "patron», pour reprendre l'expression du document ("nostro patrone »), qui illustre bien comment la relation entre les deux hommes est de nature complexe, à la fois maître-serviteur, seigneur-sujet, et 
aussi patron-employé - cette relation s'inscrit d'ailleurs dans une nomenclature administrative féodale souple, voire floue, où les appellations (sénéchal, podestat, lieutenant, etc.) semblent assez adaptables. Ce fidèle Giacomo finira par trahir son maître : le 30 janvier 1479, il dépose contre Pietro Dal Verme, et le 15 février de la même année, c'est au tour de Pietro de le dénoncer comme fugitif et comme officier indigne ${ }^{21}$.

Des agissements de Tempesta, car tel est le nom du suspect ${ }^{22}$, on ne sait pas tout, tant s'en faut : notons dès à présent le caractère lacunaire de ce dossier. On ne sait ce qu'il a fait au juste, on sait seulement, grâce aux « chapitres » dont il est question dans le texte, c'est-àdire aux différentes questions qu'il faut lui poser lors de l'interrogatoire, qu'il est soupçonné d'avoir agi contre le pouvoir ducal et contre le comte Pietro Dal Verme.

3 Ce qui est sûr est que Pietro Dal Verme l'a fait emprisonner, puis interroger par ses deux officiers, Giacomo Sabbadini et Niccolò di Castello, et que Tempesta est mort lors de l'interrogatoire. Cette déposition des deux hommes suit d'ailleurs celle de Pietro luimême, un texte adressé au duc, long de deux pages, à la suite duquel on trouve - comme pour le texte que nous traduisons - les "interrogatione et capituli », les questions qu'il faudra poser à Tempesta pendant la torture. Le témoignage que nous traduisons est plus explicite encore que celui de Pietro, car c'est celui des deux hommes qui ont mis Tempesta à la torture : ils peuvent sans intermédiaire raconter l'« accident ».

Ce récit permet tout d'abord de poser la question de la légalité du comportement des bourreaux. C'est d'ailleurs là, croyons-nous, une des raisons pour lesquelles ce texte a été produit: la légalité douteuse du décès peut expliquer que le comte et ses hommes s'expliquent sans tarder au duc. En somme, pour illégitime qu'on puisse le juger, et peu importe ici, ce meurtre est-il légal ? Notons que Pietro sort d'une période délicate sur le plan politique : sa disgrâce, entamée en 1466, et qui s'est traduite par des confiscations et par sa détention, a pris fin en 1468. Il doit donc veiller à observer un comportement qui ne déplaise pas au pouvoir ducal. Il semble bien que, l'interrogatoire étant mené à Castel San Giovanni et sur ordre du duc, les hommes de Pietro avaient le droit de procéder ainsi - leur maitre détenait là la haute justice ${ }^{23}$. La question est importante : Piero Fiorelli, dont l'ouvrage ancien demeure la référence pour ce qui concerne l'histoire de la torture, précise que torturer quand on n'en a pas le droit, par exemple quand on est un simple privé, revient à commettre un crime de lèse-majesté24. Il insiste a contrario sur le fait que la torture n'était pas nécessairement illégale; elle était encadrée, et seuls étaient répréhensibles les manquements aux règles (à tout propos: les personnes que l'on a le droit de torturer et celles qui ont le droit de le faire, le type de torture que l'on peut infliger, les crimes pour lesquels on peut le faire, etc.).

$5 \quad$ Il semble donc que la justice féodale soit ici exercée dans un cadre légal, à l'ombre de la justice ducale. Il y a assurément torture, et torture légale a priori, mais le problème est qu'elle fut mal menée : il s'agit d'une bavure. On sait en effet l'absolue nécessité faite au tortionnaire de ne pas exposer la vie de sa victime. C'est pour cela que les deux auteurs se sentent fautifs, tentent de se justifier et de se dédouaner de leur faute, qui fut de torturer Tempesta au moyen d'un système techniquement défaillant; c'est pour cela qu'ils écrivent à deux ; c'est pour cela encore que Pietro Dal Verme se remet sur eux du soin de raconter au duc (ou à ses officiers) ce qui s'est passé. Inversement, les deux hommes précisent bien que c'est Pietro qui leur a ordonné de procéder à la torture : penauds, ils se rejettent tous la faute. Ils vont même, fait extraordinaire, jusqu'à se défausser sur Dieu : n'affirment-ils pas que c'est "diuino judicio », comme par jugement divin, que la corde se rompit? Les bourreaux doivent exercer dans un cadre, où certains comportements sont 
proscrits - le sadisme ${ }^{25}$ ou, et c'est ce dont il s'agit ici, l'inconséquence. Celle-ci est doublement condamnable: non seulement parce qu'elle sort du cadre de la légalité, comme nous venons de le dire, mais aussi parce qu'elle cause du tort au seigneur qui a ordonné l'interrogatoire - n'a-t-elle pas privé Pietro et le duc des aveux qu'ils voulaient arracher à Tempesta ?

Le degré d'élaboration de la torture que pratiquent les hommes du seigneur est bien moindre que celui de la torture inquisitoriale, de la grande torture mise en œuvre contre les hérétiques. Les tortionnaires ne sont pas des lettrés, mais des officiers féodaux, assez peu cultivés ${ }^{26}$, assistés de serviteurs dont on ne sait rien. Inutile de chercher ici l'interrogatoire hautement formalisé des inquisiteurs : les bourreaux procèdent de façon bien empirique. Pour qualifier leurs actes, les auteurs des dépositions n'utilisent pas le terme usuel, "tormentum». Ils parlent d'«interrogare», d'«interrogatione» et d'" examinare ». C'est ainsi que les bourreaux considèrent leur action: une procédure juridique normale, un simple interrogatoire qui peut requérir l'emploi de ce que nous nommons torture, en dernier recours mais sans que cela ne donne lieu au moindre remords.

7 La méthode mise en œuvre pour torturer est banale ; il s'agit du supplice de la corde, qui fut pratiqué en toutes sortes de lieux et à toutes sortes d'époques, et à propos duquel Fiorelli écrit :

«[...] la principale [torture utilisée], celle qui se confondait dans le langage commun avec la torture elle-même [...], était, en Italie, dans les siècles de la Renaissance et du jus commune, le tourment de la corde [...], qui consistait à attacher les mains du patient derrière les épaules à un filin qui passait par une poulie fixée au plafond, et à le tirer en l'air, en le tenant dans cette position [...], pour ensuite, dans les cas les plus graves, le laisser retomber par terre [...]. La corde était un tourment dangereux, que l'on devait pour cette raison utiliser avec une diligence et une modération particulières; mais il présentait sur les autres tortures cet avantage, que l'on pouvait aisément le graduer, et donc l'appliquer avec une dureté plus ou moins grande en fonction de la gravité des faits, de la condition des personnes, de l'urgence des indices recueillis ${ }^{27}$."

Est bien mis en œuvre, pour torturer, un certain savoir technique, technè en grec, et ars en latin, qui fait arte en italien. Le mot « arte » apparait explicitement dans le texte, où l'on lit: «Et vedendo che né l'una arte né altra era remedio ala confessione [...]», que nous avons traduit ainsi : «Et puisque nous nous apercevions qu'aucune de ces deux techniques ne parvenait à le faire avouer [...]. » Cette traduction présente un inconvénient, celui de faire disparaitre un mot significatif, « remedio », « remède », que l'on ne peut conserver dans la traduction $^{28}$, mais qui corrobore l'idée que l'on est dans le registre de la technique, presque de la médecine. L'usage de la technique - ici corde et poulie, ailleurs tenailles ou gégène, peu importe - mêle de manière paradoxale la sophistication, l'efficacité, à la médiation de l'outil, ici la corde, qui fait qu'il n'y a pas de contact direct entre le corps du tortionnaire et celui du supplicié. La médiation est ici redoublée du fait que les donneurs d'ordre, Giacomo et Niccolò, ne sont pas les exécuteurs directs (sans oublier qu'ils ne font eux-mêmes qu'obéir à un autre donneur d'ordre, Pietro Dal Verme). Bref, la responsabilité est diluée. Reste que cette technique n'était pas maîtrisée, et que la méthode fut mal appliquée: quoi qu'ils en disent, ces tortionnaires incompétents paraissent « coupables » selon les critères juridiques qui leur sont contemporains.

9 Ce jugement de culpabilité a toutefois une limite : celle qu'impose le document qui nous permet de le formuler. Le sentiment se dégage, à la lecture du texte et contre lui, que les 
hommes ont agi de manière imprudente, incompétente, donc criminelle : mais le texte n'en demeure pas moins source unique, et évidemment instrumentalisée - il entend démontrer leur innocence. Tempesta a-t-il vraiment menti, hurlé, blasphémé ? S'est-il seulement débattu comme on le lit ? On n'en sait rien - on ne sait pas grand-chose, nous l'avons déjà dit. Le texte paraît largement codé : dans sa référence à la réalité, le texte utilise un langage riche en lieux communs, en notions qui paraissent pré-formées, en particulier pour ce qui concerne la lecture morale qui est proposée de la mort de Tempesta. Il n'est pas rare que les enjeux politiques soient redoublés, sinon masqués, par des enjeux moraux ou religieux - voir Gilles de Rais.

Sur le plan moral, plusieurs aspects frappent le lecteur, et d'abord cette manière qu'ont les tortionnaires d'utiliser des termes chrétiens pour exhorter Tempesta à avoir pitié de son propre corps : en somme, ils exercent la justice, et c'est lui, Tempesta, qui est coupable des traitements qui seraient infligés à son corps par ces deux agents de la justice. Niccolò insiste sur le fait qu'il a laissé le choix à Tempesta : « [...] je lui répétai qu'il devait nous dire la vérité car l'on aurait fait montre de miséricorde à son égard et non pas de justice, et je lui répétai de ne pas laisser abîmer sa personne ${ }^{29}$.» L'opposition entre « miséricorde » et « justice », entre douceur chrétienne et loi implacable, est significative, ainsi que la tournure qui rend Tempesta coupable de la détérioration qui pourrait être infligée à sa personne: «il devait avoir compassion de lui-même ", «il ne devait pas laisser gâter sa personne »!

11 Chrétienne encore, la dénonciation du comportement de Tempesta, peu conforme aux exigences du christianisme : il blasphème, ce qui est un manquement grave au respect dû à la religion, et ce manquement est relevé plusieurs fois. Il en appelle même au diable, à en croire le texte - forme d'hérésie discrète, mais presque attendue. Que l'hérésie affleure est très intéressant : ce n'est pas pour rien, c'est une manière de justifier l'emploi de méthodes brutales, car souvent la torture sert à lutter contre l'hérésie, avec l'approbation du pape. L'apostrophe « Diable ! » et le « jugement divin », le diable et Dieu, « diavolo » et « divino », voisinent, à quelques lignes d'écart. L'ennemi politique était donc aussi un ennemi de la religion. Comment dès lors en vouloir à nos bourreaux?

Chrétienne encore, et combien curieuse, l'expression «la corde du martyre » (« la corda del marturio ») pour désigner l'instrument de torture - ce serait toutefois surinterpréter ce mot de «martyre » que d'y voir une sorte de lapsus, comme si la torture faisait un saint de qui la subit: c'est à l'époque, en italien, et surtout dans le domaine inquisitorial, une manière parmi d'autres de désigner l'instrument de torture. L'expression demeure frappante.

Disons enfin que la connaissance du contexte politique local éclaire un peu ce cas. On apprend que l'interrogatoire de Tempesta est lié au conflit avec une famille voisine, la famille des Porro, qui a suscité de faux témoignages contre Pietro pour lui prendre le village de Corte Brugnatella, dont est originaire Tempesta. Le 21 septembre de la même année 1470, un certain Paolo Baletti avouera avoir calomnié Pietro Dal Verme à l'instigation de Tempesta, qui agissait pour Antonio Porro ${ }^{30}$. Il semble donc que Tempesta était coupable en effet : mais que croire dans ce tissu de textes dont la finalité politique est trop évidente? Il est malaisé d'avoir des certitudes; on peut du moins formuler de nombreuses hypothèses, et tenter de déchiffrer ce texte. 


\section{NOTES}

1. Galeazzo Maria Sforza, duc de Milan.

2. Cicco Simonetta, chancelier du duc de Milan, personnage de premier plan du duché. Originairede Calabre, né vers 1410, il servit Francesco Sforza dès les années 1440, avant l'accession au duché ; il servit ensuite Galeazzo Maria (1466-1476). Il fut exécuté par Ludovico il Moro à Pavie le 30 octobre 1480.

3. Bobbio, province de Plaisance, région Émilie-Romagne, fief de Pietro Dal Verme (d'abord donné en fief au père de Pietro, Luigi, par le duc de Milan Filippo Maria Visconti, en 1436).

4. Région Lombardie.

5. Le podestat du duc à Pavie ; la fonction de cet officier de l'administration régionale est surtout judiciaire.

6. Le camérier, à l'origine l'officier de la chambre d'un prélat ou d'un seigneur, est une sorte de trésorier ou d'intendant.

7. Des « galuppi », des hommes chargés de porter les bagages et de rendre divers services d'ordre matériel aux troupes.

8. Province de Plaisance, région Émilie-Romagne.

9. Région Émilie-Romagne.

10. Officier chargé de l'administration du fief.

11. Ils sont en effet transcrits à la suite de la déposition.

12. Niccolò di Castello, qui apparaît plus bas.

13. Le texte donne : «[...] el qual non respondeva altro seno ch'era impatiente.»

14. «Nél'una arte né altra », lit-on dans le texte.

15. Obietto Fieschi (1435-1497), turbulent seigneur de la région de Gênes, est alors en conflit avec les Sforza. Il sera d'ailleurs à la tête d'une révolte en 1477.

16. Ces deux souscriptions sont manifestement de deux autres mains que le reste du texte ; elles sont donc bien autographes. Le texte, lui, est de la même main que la déposition de Pietro : celle d'un troisième homme, un scribe anonyme qui a rédigé les deux dépositions.

17. Les passages en latin sont en italique dans notre traduction.

18. Archivio di Stato di Milano, Sforzesco, 850. Les Sforza sont ducs de Milan dans la seconde moitié du XVe siècle (Francesco Sforza, 1450-1466 ; Galeazzo Maria Sforza, 1466-1476 ; enfin Gian Galeazzo et Ludovico Sforza, ou Ludovico il Moro). Ils succèdent, après l'intermédiaire de la République ambrosienne (1447-1450), aux Visconti, seigneurs de Milan depuis la fin du XIII ${ }^{\mathrm{e}}$ siècle, ducs de Milan de 1395 à 1447.

19. On peut distinguer entre ces deux aspects de la torture. Sur le second, la torture punitive, voir Nicole Gonthier, Le Châtiment du crime au Moyen Âge. XII ${ }^{e}-\mathrm{XVI}^{e}$ siècles, Rennes, 1998.

20. Province de Varese, région Lombardie.

21. Archivio di Stato di Milano, Finanze - confische, 3164, et Sforzesco, 858.

22. Tempesta doit être son prénom; il ne semble pas avoir de nom défini. On le définit par sa provenance géographique : Tempesta de Bobbio, ou de Brugnadello (aujourd'hui Corte Brugnatella, province de Plaisance, région Émilie-Romagne). 
23. La haute justice concerne les crimes les plus graves : crimes de sang, trahison, etc. Les Dal Verme la détiennent en vertu de l'acte par lequel Castel San Giovanni est donné en fief à Luigi Dal Verme, en même temps que Bobbio (voir supra).

24. Piero Fiorelli, La Tortura giudiziaria nel diritto comune, Milan, 1953-1954, 2 vol., 2, p. 176, qui donne à la note 4, pp. 176-177, quelques exemples de seigneurs tombant dans l'illégalité car mettant un homme à la torture, etc. Voir aussi, sur la torture, dans une bibliographie souvent nauséabonde, Edward Peters, Torture, New York et Oxford, 1985, surtout le chapitre 2, "The Queen of Proofs and the Queen of Torments »; et un ouvrage plus général, Trevor Dean et Kate J. P. Lowe (sous la direction de), Crime, Society and the Law in Renaissance Italy, Cambridge, 1994.

25. Voir dans ce numéro la contribution de Stéphanie Genand, « Entre asservissement sexuel et despotisme politique. La figure du bourreau chez Sade ».

26. Notons que Sabbadini, à en juger par sa souscription, écrit fort mal.

27. Piero Fiorelli, op. cit., 2, pp. 194-196.

28. Sauf à accepter « aucune de ces deux techniques n'était un remède permettant l'aveu...».

29. «[...] repplicai volesse dire el vero che li saria usato misericordia et non justicia et non se volesse lasare guastare la persona. »

30. Archivio di Stato di Verona, Archivio Zileri Dal Verme, Perg., Perg., 424 et 425.

\section{AUTEUR}

\section{PIERRE SAVY}

Pierre Savy est né à Paris en 1975. Ancien élève de l'Ėcole normale supérieure et agrégé d'histoire, il est membre de l'Ėcole française de Rome depuis le mois de septembre 2002 et prépare un doctorat d'histoire sous la direction de Bertrand Schnerb sur la féodalité dans le duché de Milan au Xv $\mathrm{v}^{\mathrm{e}}$ siècle. Il est, avec David Schreiber, directeur de la rédaction de Labyrinthe. 